\title{
Local Structure of Riemannian Manifolds
}

\section{Patrick Ghanati, Maung Min-Oo $\mathscr{G}$ Ernst A. Ruh}

\begin{abstract}
We show that small neighborhoods of points in a Riemannian manifold equipped with an orthonormal coframe are diffeomorphic to products of euclidean balls and nilmanifolds. The size of these neighborhoods is uniformly bounded in terms of the dimension of the manifold and the exterior derivative of the coframe.
\end{abstract}

In this paper we prove that a Riemannian manifold $M$ equipped with an orthonormal coframe $\omega: T M \rightarrow \mathbb{R}^{n}$ locally looks like a product of a (closed) nilmanifold and a euclidean ball, if considered on a suitable scale. Every point in $M$ has a neighborhood diffeomorphic, in fact almost affinely isometric to such a product, and containing a distance ball whose radius is bounded from below in terms of the size $\|d \omega\|$ of the exterior derivative of $\omega$ and the dimension $n$ of $M$. In particular, if $M$ is closed, connected and has small diameter, then $M$ is diffeomorphic to a nilmanifold and we obtain the main result in [Gh]. Results similar to our theorem have been obtained by K. Fukaya [F1] from a different point of view. This paper was completed while the first and third authors were staying at and supported by the Forschungsinstitut für Mathematik at ETH Zürich. We would like to thank Professor J. Moser and the Forschungsinstitut for their support and hospitality.

\section{Statement of results.}

1.1. Let $M$ denote an $n$-dimensional $C^{\infty}$ manifold and $\omega: T M \rightarrow \mathbb{R}^{n}$ a coframe. This means that $\omega$ is an $\mathbb{R}^{n}$-valued one-form that maps each tangent space $T_{p} M$ isomorphically onto $\mathbb{R}^{n}$. Let $X_{1}, \ldots, X_{n}$ be the frame dual to $\omega, D$ the flat connection on $T M$ defined by $D X_{i}=0(i=1, \ldots, n)$ and $\exp =\exp ^{D}$ the exponential map of $D$. The geodesics of $D$ are the integral curves of linear combinations of the vector fields $X_{i}$ with constant coefficients. Let $g=g_{\omega}=$ $\sum_{i=1}^{n} \omega^{i} \otimes \omega^{i}$ denote the Riemannian metric induced by $\omega$, making $X_{1}, \ldots, X_{n}$ 
orthonormal. Let $\kappa=\|d \omega\|_{\infty}$, where $\|\cdot\|_{\infty}$ is the sup norm induced by $g$. For a submanifold $N \subseteq M$ with normal bundle $\nu$ and $\rho>0$ let

$$
\nu_{\rho}=\{X \in \nu \mid\|X\|<\rho\}
$$

and $\exp ^{\perp}=\left.\exp ^{D}\right|_{\nu}$ the normal exponential map.

Theorem 1.2. There is a positive constant $\varepsilon(n)$ depending only on $n$ and a function $\delta: \mathbb{R} \rightarrow \mathbb{R}$ satisfying $\lim _{t \rightarrow 0} \delta(t)=0$ such that the following is true. Suppose $\varepsilon_{1}>0$ and $0 \leq \kappa \varepsilon_{1} \leq \varepsilon(n)$. If $p \in M$ and if $\exp$ is defined on the ball $B\left(0,10 \varepsilon_{1}\right) \subseteq T_{p} M$, then there exists a radius $R$ such that $10^{-n} \varepsilon_{1} \leq R \leq \varepsilon_{1}$ and such that the distance ball $B(p, R) \subseteq M$ contains a nilmanifold $N^{m} \ni p . N$ is embedded in $M$ with trivial normal bundle $\nu$ and the normal exponential map $\exp ^{\perp}$ of $N$ maps $\nu_{2 R}$ diffeomorphically onto a neighborhood of $B(p, R)$. There is a product Maurer-Cartan coframe $\omega_{0}$ on $\nu_{2 R} \simeq N \times D_{2 R}$ and a matrix $a \in S O(n)$ such that $\left\|\exp _{*}^{\perp} \omega_{0}-\omega \cdot a\right\|_{\infty} \leq \delta\left(\kappa \varepsilon_{1}\right)$ on $\exp ^{\perp}\left(\nu_{2 R}\right)$.

Here $D_{2 R}$ denotes the open ball of radius $2 R$ in $\mathbb{R}^{k}(k+m=n)$ and $B(p, R)=\{q \in M \mid \operatorname{dist}(p, q)<R\}$. We recall that a nilmanifold is a compact quotient $\Gamma \backslash G$ of a simply connected nilpotent Lie group $G$ by a discrete subgroup $\Gamma$. A Maurer-Cartan coframe on a smooth manifold $M$ is a smooth coframe $\omega: T M \rightarrow \mathbb{R}^{n}$ whose component 1 -forms $\omega^{1}, \ldots, \omega^{n}$ satisfy the MaurerCartan equations $d \omega^{s}+\frac{1}{2} c_{i j}^{s} \omega^{i} \wedge \omega^{j}=0(s=1, \ldots, n)$ where the $c_{i j}^{s}$ are constants. The product Maurer-Cartan form $\omega_{0}$ in the theorem consists of a coframe $\eta_{0}: N=\Gamma \backslash G \rightarrow \mathbb{R}^{m}$ whose pullback to $G$ is left invariant, and the one-forms $d x^{1}, \ldots, d x^{k}$ on $D_{2 R}$. We note that no compactness arguments in the style of [F1] are used in the proof of 1.2. As a consequence, the constants $\varepsilon(n)$ and functions $\delta(t)$ in 1.2 and 1.3 are effective. However, no explicit bounds will be given in this paper.

1.2.1. If $M$ is compact and the diameter $d=\operatorname{diam}\left(M, g_{\omega}\right)$ satisfies $\kappa d \leq$ $10^{-n} \varepsilon(n)$, then 1.2 implies that $m=n$ and $M$ is diffeomorphic to the nilmanifold $\Gamma \backslash G=N$. This special case of 1.2 was obtained previously in [Gh] and will be used in the proof.

1.3. Let $M^{n}$ be a Riemannian manifold and $P$ its bundle of orthonormal frames. Then $P$ carries a coframe $\omega=\vartheta+\alpha$ where $\alpha: T P \rightarrow s o(n)$ is the LeviCività connection form and $\vartheta: T P \rightarrow \mathbb{R}^{n}$ the canonical one-form. Consider the standard euclidean scalar product on $\mathbb{R}^{n}$ and the negative of the CartanKilling form on $s o(n)$. Then the Riemannian metric $g_{\omega}$ on $P$ induced by $\omega$ is the standard (Sasaki) metric making the projection $P \rightarrow M$ a Riemannian submersion. The structure equations for $d \vartheta$ and $d \alpha$ together with $1.2 \mathrm{imply}$ the following stronger version of a result originally announced by J. Bemelmans and the third author in 1985 ([B-R]). 
Corollary 1.3.1. There exist a positive constant $\varepsilon(n)$ and a function $\delta$ : $\mathbb{R} \rightarrow \mathbb{R}$ satisfying $\lim _{t \rightarrow 0} \delta(t)=0$ such that the following is true. If $M$ is a complete Riemannian manifold with sectional curvature $|K| \leq 1$ and if $\varepsilon_{1} \leq \varepsilon(n)$, then every distance ball $B \subseteq P$ of radius $\varepsilon_{1}$ admits an equidimensional imbedding $\varphi: B \rightarrow \mathbb{R}^{k} \times \Gamma \backslash G$ where $\Gamma \backslash G$ is a nilmanifold, such that $\left\|\varphi^{*} \omega_{0}-\omega\right\|_{\infty}<\delta\left(\varepsilon_{1}\right)$ for a quotient $\omega_{0}$ of some left invariant coframe on $\mathbb{R}^{k} \times G$.

More precise statements on the structure of $P$ and $M$ can be made. This will be the subject of a forthcoming article.

Remark 1.3.2. According to the Hirsch immersion theorem (see $[\mathrm{H}],[\mathrm{G} 2]$ ), for any coframe $\omega$ on an open manifold $M^{n}$ there exists a curve $\omega_{t}(0 \leq t \leq 1)$ of coframes on $M$ such that $\omega_{0}=\omega$ and $\omega_{1}=d \psi: M \rightarrow \mathbb{R}^{n}$. In particular, $\omega_{1}$ solves the abelian Maurer-Cartan equation $d \omega_{1}=0$. By contrast, 1.2 assumes smallness of $d \omega$ and yields a nilpotent Maurer-Cartan form $\omega_{1}$ close to $\omega$. Also, 1.2 applies to closed manifolds (1.2.1) for which the immersion theorem fails.

The nilmanifold $N$ in 1.2 depends on the choice of $p$ as well as $\varepsilon_{1}$. Interesting examples are obtained by collapsing ([F2]).

Example 1.4 (see [G1]). Let $\Gamma \backslash G$ be a nilmanifold. Thus $G$ is a simply connected nilpotent Lie group and $\Gamma \leq G$ a lattice. Let $g$ be an inner product on the Lie algebra $\mathcal{G}$ of $G$. Then $g$ induces a left invariant Riemannian metric on $G$ that descends to a metric on $\Gamma \backslash G$ which we also denote by $g$. Let $\mathcal{G}=\mathcal{G}^{(0)} \geq$ $\mathcal{G}^{(1)} \geq \ldots \geq \mathcal{G}^{(r)}=\{0\}$ denote the descending central series and let $U_{k}$ denote the orthogonal complement of $\mathcal{G}^{(k+1)}$ in $\mathcal{G}^{(k)}$, so that $\mathcal{G}^{(k)}=U_{k} \oplus \mathcal{G}^{(k+1)}$. Then we have a decomposition $\mathcal{G}=U_{0} \oplus U_{1} \oplus \cdots \oplus U_{r-1}$ into orthogonal subspaces. Since $\left[\mathcal{G}^{(i)}, \mathcal{G}^{(j)}\right] \subseteq \mathcal{G}^{(i+j+1)}$, one gets

$$
\left[U_{i}, U_{j}\right] \subseteq \bigoplus_{k \geq i+j+1} U_{k}
$$

Choose numbers $\lambda_{0}, \ldots, \lambda_{r-1}$ such that $\lambda_{i+j+1} \leq \lambda_{i} \lambda_{j}$ and multiply $g$ by $\lambda_{i}$ on $U_{i}$ to obtain a new inner product $g_{\lambda}$. Consider left invariant coframes $\omega_{\lambda}$ orthogonal with respect to $\mathcal{G}_{\lambda}$. Since $\left\|d \omega_{\lambda}\right\|=\kappa_{\lambda}$ is the norm of the Lie bracket on $\mathcal{G}, 1.4 .1$ implies that $\kappa_{\lambda} \leq$ const as $\lambda \rightarrow 0$. Depending on the choice of $\lambda,\left(\Gamma \backslash G, g_{\lambda}\right)$ converges in the Hausdorff sense to a point or a lower dimensional nilmanifold. Let $G=G^{(0)} \geq G^{(1)} \geq \ldots \geq G^{(r)}=1$ denote the descending central series of $G$. For $\lambda$ small enough there are $\varepsilon_{1}$ such that $\kappa_{\lambda} \varepsilon_{1} \leq \varepsilon(n)$ and the nilmanifold $N$ of 1.2 is any one of the quotients $G^{(i)} / G^{(i)} \cap \Gamma(0 \leq i \leq r)$, depending on the choice of $\varepsilon_{1}$. 


\section{The proof.}

2.1. We use the notation introduced in 1.1. A simple scaling argument shows that one can assume $\kappa \leq \varepsilon_{2}(n)$ where $\varepsilon_{2}$ is an arbitrary small constant whose size will be fixed later in the proof and depends only on $n$. In fact, if $\varepsilon_{1}$ is chosen such that $\kappa \varepsilon_{1} \leq \varepsilon(n)$, consider the coframe $\omega_{\lambda}=\lambda \omega(\lambda>0)$ on $M$. Denoting quantities defined using $\omega_{\lambda}$ by a subscript $\lambda$, one checks $\kappa_{\lambda}=\frac{\kappa}{\lambda}$ and for distance balls with respect to $g_{\lambda}, B_{\lambda}(p, \rho)=B\left(p, \frac{\rho}{\lambda}\right)$. Let $\varepsilon_{1}^{\prime}=\lambda \varepsilon_{1}$. Then $\varepsilon_{1}^{\prime} \kappa_{\lambda}=\varepsilon_{1} \kappa \leq \varepsilon(n)$ and $B_{\lambda}\left(p, \varepsilon_{1}^{\prime}\right)=B\left(p, \varepsilon_{1}\right)$. If 1.2 is proven with the additional hypothesis $\kappa \leq \varepsilon_{2}(n)$, we can choose $\lambda$ large and apply 1.2 to $\omega_{\lambda}$. But then 1.2 follows for $\omega$ itself (with a rescaled $\omega_{0}$ ).

2.2. The exponential map $\exp =\exp _{p}^{D}$ at $p \in M$ satisfies the estimate $([\mathrm{Gh}])$

$$
\left(2-e^{\kappa\|x\|}\right)\|Y\| \leq\left\|d \exp _{x} Y\right\| \leq e^{\kappa\|x\|} \cdot\|Y\|
$$

for $x$ in its domain and $Y \in T_{x} T_{p} M \simeq T_{p} M$. Therefore, by choosing $\varepsilon(n)$ small exp will be nearly a local isometry on $B\left(0,10 \varepsilon_{1}\right)$. Let $\bar{\omega}, \bar{g}$ and $\bar{D}$ denote the pullbacks of $\omega, g$ and $D$ to $B\left(0,10 \varepsilon_{1}\right)$ under exp. The components of $\bar{\omega}^{i}$ of $\bar{\omega}$ satisfy $([\mathrm{Gh}])$

$$
\left\|\bar{\omega}^{i}(x)-d x^{i}\right\| \leq\left(2-e^{k\|x\|}\right)^{-1}\left(e^{\kappa\|x\|}-1\right)
$$

for $x \in B\left(0,10 \varepsilon_{1}\right)$, where the $x^{i}$ are linear coordinates on $T_{p} M$ such that $d x^{i}=\bar{\omega}^{i}$ at the origin and the norm is taken with respect to the euclidean metric $g(p)$ on $T_{p} M$.

2.3. For $\rho \leq 10 \varepsilon_{1}$ let $\Gamma_{\rho}=\exp ^{-1}(p) \cap B(0, \rho) \subseteq T_{p} M$. For $3 \rho \leq 10 \varepsilon_{1}$ each $x \in \Gamma_{\rho}$ defines an imbedding $\gamma_{x}: B(0, \rho) \rightarrow B(0,3 \rho)$ characterized by $\gamma_{x}(0)=x$ and expo $\gamma_{x}=\exp$. Since $\gamma_{x}^{*} \bar{\omega}=\bar{\omega}, 2.2$ implies that $\gamma_{x}$ is $C^{1}$-close to the translation by $x$. We also note that $\gamma_{x}$ leaves the line $\mathbb{R} x$ invariant and acts by translation on that line.

Let $x_{1}, x_{2} \in B(0, \rho)$. Then $\exp \left(x_{1}\right)=\exp \left(x_{2}\right)$ if and only if there exists $x \in \Gamma_{3 \rho}$ such that $\gamma_{x}\left(x_{1}\right)=x_{2}$. In fact, let $U \subseteq T_{p} M$ be a neighborhood of $x_{2}$ such that exp restricted to $U$ is an imbedding. Define $\gamma$ near $x_{1}$ by $\gamma=$ $\left(\left.\exp \right|_{U}\right)^{-1}$ o exp and extend $\gamma$ to a map $\gamma: B(0, \rho) \rightarrow B(0,3 \rho)$ by mapping geodesics of $\bar{D}$ starting at $x_{1}$ to corresponding geodesics starting at $x_{2}$. Here we are using the fact that by 2.2 the exponential map $\exp ^{\bar{D}}$ of the pullback connection $\bar{D}$ has derivative close to the identity and therefore large injectivity radius. Let $x=\gamma(0) \in B(0,3 \rho)$. Since $\exp \circ \gamma=\exp$, it follows that $\gamma=\gamma_{x}$. Note that $x$ almost coincides with $x_{2}-x_{1}$. 
2.4. In the rest of the proof, $\delta_{i}(i=1,2,3, \ldots)$ will denote functions $\mathbb{R} \rightarrow \mathbb{R}^{+}$ such that $\lim _{t \rightarrow 0} \delta_{i}(t)=0$. For a subset $V \subseteq T_{p} M$ and $r>0$ let $V(r)$ denote the euclidean $r$-neighborhood $V(r)=\left\{x \in T_{p} M \mid \operatorname{dist}(x, V)<r\right\}$. In several places we will omit the phrase "if $\varepsilon(n)$ is chosen small enough."

Lemma 2.5. There is a $\rho$ such that $10^{-n} \varepsilon_{1} \leq \frac{\rho}{9} \leq \varepsilon_{1}$ and a subspace $V \subseteq T_{p} M$ such that

(1) $\Gamma_{\rho} \subseteq V\left(\delta_{1}\left(\kappa \varepsilon_{1}\right)\right)$,

(2) $\forall x \in V \cap B(0, \rho): \operatorname{dist}\left(x, \Gamma_{\rho}\right)<\frac{\rho}{9}$.

The notation in (1) is explained in 2.4, and dist denotes the eucloidean distance in $T_{p} M$. Eventually, $R=\frac{\rho}{9}$ will be chosen for the radius $R$ in 1.2.

Proof. We first show that there exist $\rho$ and $V$ such that $(1)^{\prime}$ and (2) hold where $(1)^{\prime} \Gamma_{\rho} \subseteq V\left(\frac{\rho}{10}\right)$.

To see this, let $\rho_{0}=10^{-n} 9 \varepsilon_{1}$. If $\Gamma_{\rho_{0}}=\{0\}$, choose $V=\{0\}$ and $\rho=\rho_{0}$. Otherwise pick $x_{1} \in \Gamma_{\rho_{0}}$ not equal to zero, let $V_{1}=\operatorname{span}\left\{x_{1}\right\}$ and $\rho_{1}=10 \rho_{0}$. Define $I_{1}=\left\{k \in \mathbb{Z} \mid \gamma_{x_{1}}^{\ell}(0) \in B\left(0, \rho_{1}\right)\right.$ for all $\ell$ between 0 and $\left.k\right\}$ and define the orbit $\mathcal{O}_{1}=\left\{\gamma_{x_{1}}^{k}(0) \mid k \in I_{1}\right\} \subseteq \Gamma_{\rho_{1}} \cap V_{1}\left(\rho_{0}\right)$. Clearly $\mathcal{O}_{1}$ satisfies $\operatorname{dist}\left(x, \mathcal{O}_{1}\right) \leq$ $\rho_{1} / 9$ for all $x \in V_{1}$. If $\Gamma_{\rho_{1}} \subseteq V_{1}\left(\rho_{0}\right)$, then (1)' and (2) hold for $V=V_{1}$ and $\rho=\rho_{1}$. Otherwise choose $x_{2} \in \Gamma_{\rho_{1}}$ outside $V_{1}\left(\rho_{0}\right)$, define $V_{2}=\operatorname{span}\left\{x_{1}, x_{2}\right\}$ and $\rho_{2}=10 \rho_{1}$. Define $I_{2}=\left\{\left(k_{1}, k_{2}\right) \in \mathbb{Z} \times \mathbb{Z} \mid \gamma_{x_{1}}^{\ell_{1}} \gamma_{x_{2}}^{\ell_{2}}(0) \in B\left(0, \rho_{2}\right)\right.$ for all $\ell_{1}$ between 0 and $k_{1}$ and all $\ell_{2}$ between 0 and $\left.k_{2}\right\}$. Define the orbit $\mathcal{O}_{2}=$ $\left\{\gamma_{x_{1}}^{k_{1}} \gamma_{x_{2}}^{k_{2}}(0) \mid\left(k_{1}, k_{2}\right) \in I_{2}\right\} \subseteq \Gamma_{\rho_{2}} \cap V_{2}\left(\rho_{1}\right)$. Then $\operatorname{dist}\left(x, \mathcal{O}_{2}\right) \leq \rho_{2} / 9$ for all $x \in V_{2}$. If $\Gamma_{\rho_{2}} \subseteq V_{2}\left(\rho_{1}\right)$, then (1)' and (2) hold for $V=V_{2}$ and $\rho=\rho_{2}$. Otherwise choose $x_{3} \in \Gamma_{\rho_{2}}$ outside $V_{2}\left(\rho_{1}\right)$, define $V_{3}=\operatorname{span}\left\{x_{1}, x_{2}, x_{3}\right\}$ and $\rho_{3}=10 \rho_{2}$ and continue. Since $\operatorname{dim}\left(V_{i}\right)=i$ the procedure terminates after $m \leq n$ steps. Set $V=V_{m}$ and $\rho=\rho_{m}$.

Note that $m=0$ means that exp has large injectivity radius and corresponds to the case $N^{m}=\{p\}$ of the theorem. If $m=n, M$ is compact with small diameter and 1.2.1 can be applied. Finally, we claim that $V$ and $\rho$ obtained above satisfy (1). If not, we could use the almost-translations $\gamma_{x}, x \in \mathcal{O}_{m}$ repeatedly to produce an element in $\Gamma_{\rho}$ outside $V\left(\frac{\rho}{10}\right)$. The argument actually shows that $\left\|x^{\perp}\right\| \leq \delta_{2}\left(\kappa \varepsilon_{1}\right) \cdot\|x\|$ for the $V^{\perp}$-component $x^{\perp}$ of any element $x \in \Gamma_{\rho}$.

2.6. After rotating the coframe $\omega$ by a constant matrix $a \in S O(n)$ we may assume that $V=\operatorname{span}\left\{X_{1}(p), \ldots, X_{m}(p)\right\}$. Recall that linear coordinates are chosen on $T_{p} M$ such that $X_{i}(p)=\partial /\left.\partial x^{i}\right|_{0}$. In the following we construct a closed almost integral manifold $N^{m}$ of the subbundle of $T M$ spanned by $X_{1}, \ldots, X_{m}$. $N^{m}$ will turn out to be a nilmanifold. 
2.7. Let $\mu: T_{p} M \rightarrow[0,1]$ be smooth and such that $\mu=1$ on $B\left(0, \frac{\rho}{2}\right), \mu=0$ outisde $B(0, \rho)$ and $\|d \mu\|<\frac{2.9}{\rho}$. Let $\varphi^{s}=x^{m+s}(s=1, \ldots, k)$ denote the last $k$ coordinate functions on $T_{p} M$, where $k=n-m$. Define for $x \in B(0, \rho)$

$$
\bar{f}^{s}(x)=\frac{\sum \mu\left(x^{\prime}\right) \varphi^{s}\left(x^{\prime}\right)}{\sum \mu\left(x^{\prime}\right)} \quad(s=1, \ldots, k)
$$

where the sums are taken over all $x^{\prime} \in B(0, \rho)$ such that $\exp \left(x^{\prime}\right)=\exp (x)$. By definition of $\bar{f}^{s}$, there exists a smooth function $f^{s}$ on the open subset $U_{\rho}:=$ $\exp B(0, \rho) \subseteq M$ such that $f^{s} \circ \exp =\bar{f}^{s}$. We estimate

$$
\left|\bar{f}^{s}(x)-\varphi^{s}(x)\right| \leq \max \left|\varphi^{s}\left(x^{\prime}\right)-\varphi^{s}(x)\right| \leq \delta_{1}\left(\kappa \varepsilon_{1}\right)
$$

Let $\bar{X}_{i}$ denote the lift of $X_{i}$ to $B\left(0,10 \varepsilon_{1}\right)$ under exp. In order to compute the directional derivatives of $\bar{f}^{s}$ with respect to $\bar{X}_{i}$ we write $x^{\prime}=\gamma x$ in the definition of $\bar{f}^{s}$ (see 2.3) and extend the sum over the corresponding $\gamma$ 's. Since $\gamma_{*} \bar{X}_{i}=\bar{X}_{i}$, one obtains

$$
\begin{aligned}
\left(\bar{X}_{i} \bar{f}^{s}\right)(x)= & \left(\sum \mu\left(x^{\prime}\right)\right)^{-1} \bar{X}_{i}\left(\sum \mu(\gamma x) \varphi^{s}(\gamma x)\right) \\
& -\bar{f}^{s}(x)\left(\sum \mu\left(x^{\prime}\right)\right)^{-1} \bar{X}_{i}\left(\sum \mu(\gamma x)\right) \\
= & \left(\sum \mu\left(x^{\prime}\right)\right)^{-1} \sum\left(\bar{X}_{i} \mu\right)\left(x^{\prime}\right) \cdot\left(\varphi^{s}\left(x^{\prime}\right)-\bar{f}^{s}(x)\right) \\
& +\left(\sum \mu\left(x^{\prime}\right)\right)^{-1} \sum \mu\left(x^{\prime}\right)\left(\left(\bar{X}_{i} \varphi^{s}\right)\left(x^{\prime}\right)-\delta_{i}^{m+s}\right)+\delta_{i}^{m+s}
\end{aligned}
$$

By (2.2.2) we have $\left|\bar{X}_{i} \mu\right|=\left|d \mu\left(\bar{X}_{i}\right)\right| \leq \frac{3}{\rho}$ if $\varepsilon(n)$ is chosen small enough. Let $A(X, \rho)$ denote the number of points $x^{\prime}$ inside $B(0, \rho)$. Then, using (2.7.1) and $(2.2 .2)$,

$$
\left|\left(\bar{X}_{i} \bar{f}^{s}\right)(x)-\delta_{i}^{m+s}\right| \leq \frac{3}{\rho} \delta_{1}\left(\kappa \varepsilon_{1}\right) \frac{A(x, \rho)}{A(x, \rho / 2)}+\delta_{3}\left(\kappa \varepsilon_{1}\right) .
$$

Since $\frac{A(x, \rho)}{A(x, \rho / 2)} \leq c(n)$, we obtain on $B(0, \rho)$

$$
\left\|d \bar{f}^{s}-d x^{m+s}\right\| \leq \frac{1}{\rho} \delta_{4}\left(\kappa \varepsilon_{1}\right)+\delta_{5}\left(\kappa \varepsilon_{1}\right)
$$

Since $\rho \geq 10^{-n} \varepsilon_{1}$ and $\kappa \leq \varepsilon_{2}(n)$ (see 2.1), the right-hand side of (2.7.2) will be small if $\varepsilon(n)$ was chosen small enough. As a result, $\bar{f}^{s}$ is $C^{1}$-close to the coordinate function $x^{m+s}$. 
2.8. Define $N \subseteq M$ by $N=\left\{q \in U_{\rho} \mid f^{s}(q)=f^{s}(p)\right.$ for $\left.s=1, \ldots, k\right\}$. By 2.7, $N$ is an $m$-dimensional submanifold of $U_{\rho}$ without boundary, equal to $\exp (\bar{N})$ where

$$
\bar{N}=\left\{x \in B(0, \rho) \mid \bar{f}^{s}(x)=\bar{f}^{s}(0) \text { for } s=1, \ldots, k\right\} .
$$

The vector fields $X_{1}, \ldots, X_{m}$ are almost tangent to $N$ because of 2.7. $\bar{N}$ is diffeomorphic to the open $m$-ball. Therefore, $N$ is connected. $N$ is compact. In fact, if $p_{i} \in N$ is a sequence in $N$, we can choose $x_{i} \in B\left(0, \frac{\rho}{9}\right)$ such that $\exp \left(x_{i}\right)=p_{i}$. Then the sequence $\left\{x_{i}\right\}$ has a convergent subsequence. Clearly $N$ has trivial normal bundle. Let $\iota: N \rightarrow M$ denote the inclusion. $N$ has diameter bounded above by $\frac{\rho}{9}$ with respect to the induced metric $\iota^{*} g$. The metric $\iota^{*} g$ coincides with the Riemannian metric induced by the coframe $\eta$ : $T N \rightarrow \mathbb{R}^{m}, \eta^{i}=\iota^{*} \omega^{i}(i=1, \ldots, m)$. Since $d \eta^{i}=\iota^{*} d \omega^{i}, \eta$ satisfies the hypothesis $\operatorname{diam}\left(g_{\eta}\right) \cdot\|d \eta\|_{\infty}<\varepsilon_{3}(n)$ of the main result in [Gh] (compare 1.2.1). It follows that $N$ is a nilmanifold and there is a Maurer-Cartan coframe $\eta_{0} C^{0}$-close to $\eta$ on $N$.

2.9. It remains to prove the statements concerning the normal exponential map $\exp ^{\perp}$ of $N . \exp ^{\perp}$ is obtained by integrating parallel vector fields orthogonal to $N$. Since $\exp _{p}$ is defined on $B\left(0,10 \frac{\rho}{9}\right)$, $\exp ^{\perp}$ is defined on $\nu_{\rho}=\{X \in \nu \mid\|x\|<$ $\rho\}$. Standard Jacobi field estimates (compare [Gh]) show that $\exp ^{\perp}$ has maximal rank on $\nu_{\rho}$ and maps $\nu_{2 \rho / 9}$ onto a neighborhood of $B\left(p, \frac{\rho}{9}\right)$.

We show that $\exp ^{\perp}$ is injective on $\nu_{2.1 \rho / 9}$. If not, then there exist points $x_{1}$, $x_{2} \in \bar{N}$ such that $\operatorname{dist}\left(x_{1}, 0\right)<\frac{\rho}{9}$ and $\operatorname{dist}\left(x_{1}, x_{2}\right)<\frac{\rho}{9}$ and $\bar{D}$-geodesics $c_{1}$ and $c_{2}$ starting at $x_{1}$ and $x_{2}$, respectively, of $\bar{g}$-length less that $\frac{2.1 \rho}{9}$ and orthogonal to $\bar{N}$, whose endpoints $y_{1}$ and $y_{2}$ satisfy $\exp \left(y_{1}\right)=\exp \left(y_{2}\right)$. Since $\operatorname{dist}\left(y_{1}, y_{2}\right)<\frac{5.3 \rho}{9}$, there exists $x \in \Gamma_{5.4 \rho / 9}$ such that $\gamma_{x}\left(y_{1}\right)=y_{2} . \gamma_{x}$ preserves $\bar{\omega}$ and therefore maps $c_{1}$ into a geodesic $c_{1}^{\prime}$ joining some point $x_{1}^{\prime} \in \bar{N} \cap B\left(0,6.5 \frac{\rho}{9}\right)$ to $y_{2}$. The curve $c_{1}^{\prime}$ is orthogonal to $\bar{N}$ at $x_{1}^{\prime}$. However, since $\bar{N}$ is $C^{1}$-close to a subspace $V$ and $\bar{\omega}$ is $C^{0}$-close to $d x$, the normal exponential map $\exp _{\bar{N}}^{\perp}$ of $\bar{N}$ with respect to $\bar{D}$ has injectivity radius greater than $2.1 \frac{\rho}{9}$. It follows that $x_{1}^{\prime}=x_{2}$ and $c_{1}^{\prime}=c_{2}$. This proves the injectivity of $\exp ^{\perp}$ on $\nu_{2.1 \rho / 9}$.

For 1.2 we choose $R=\frac{\rho}{9}$. The trivialization $\Phi: N \times D_{2 R} \rightarrow \nu_{2 R}$ is given by sections $X_{m+1}^{\prime}, \ldots, X_{n}^{\prime}$ of $\nu C^{0}$-close to $X_{m+1}, \ldots, X_{n}$. The product MaurerCartan form $\omega_{0}$ on $N \times D_{2 R}$ can be described as $\omega_{0}=\pi_{1}^{*} \eta_{0} \oplus \pi_{2}^{*} d x$ where $\pi_{1}$ and $\pi_{2}$ are the projections from $N \times D_{2 R}$ onto the factors $N$ and $D_{2 R}$, respectively. It is not difficult to see that $\Phi$ maps $\omega_{0}$ into a coframe $C^{0}$-close to the pullback of $\omega \cdot a$ onto $\nu_{2 R}$ under the normal exponential map $\exp ^{\perp}$. Recall that $\omega$ was rotated by $a \in S O(n)$ in 2.6. This finishes the proof of the theorem. 


\section{REFERENCES}

[B-R] J. Bemelmans 6 E. A. RuH, The local structure of Riemannian manifolds, Abstracts of Papers presented to the Amer. Math. Soc. 6, 816-53-303. January 1985

[F1] K. FUKAYA, Hausdorff convergence of Riemannian manifolds and its application, (Preprint) .

[F2] K. FUKAYA, Collapsing Riemannian manifolds to ones with lower dimensions, II, J. Math. Soc. Japan 41 (1989), 333-356.

[Gh] P. GhanaAt, Almost Lie groups of type $\mathbb{R}^{n}$, J. reine angew. Math. 401 (1989), 60-71.

[G1] M. Gromov, Almost flat manifolds, J. Differential Geometry 13 (1978), 231-241.

[G2] M. Gromov, Partial Differential Relations, Ergebnisse der Math. 9. Berlin: Springer-Verlag, 1986 .

[H] M. HiRsch, On imbedding differential manifolds into euclidean space, Ann. Math. 73 (1961), 566-571.

[R] E. A. RuH, Almost flat manifolds, J. Differential Geometry 17 (1982), 1-14.

Patrick Ghanaat's research was partially supported by the Forschungsinstitut für Mathematik at ETH Zürich. Maung Min-Oo's research was partially supported by N. S. E. R. C. Grant A7873 of Canada. Ernst A. Ruh's research was partially supported by the Forschungsinstitut für Mathematik at ETH Zürich and N. S. F. Grant DMS-8601282 of the USA.

\section{PATRICK GHANAAT}

Mathematisches Institut

Universität Basel

Rheinsprung 21

CH-4051 Basel

Switzerland

Received: July 5, 1990.

\author{
MAUng Min-Oo \\ Department of Mathematics \\ McMaster University \\ Hamilton, Ontario \\ Canada L8S 4K1
}

ERnst A. RuH

Department of Mathematics The Ohio State University 231 West 18th Avenue Columbus, Ohio 43210 